\title{
Liberación de delta-ala en orina de niños: un indicador de contaminación por plomo en Santiago
}

\author{
(Urine concentration of delta ALA in children: an \\ indicator of lead centamination in Santiago!
}

Dr. Rubén Cisternas Y., Srta. Múnica Sáez F.

\begin{abstract}
ABSTR ALT

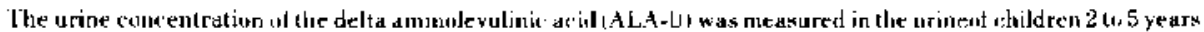

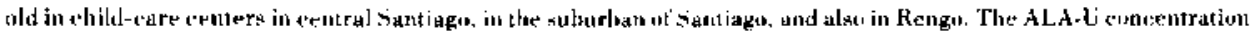

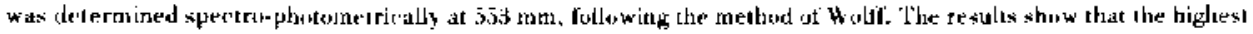

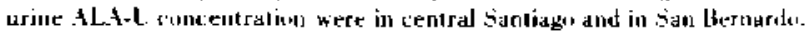

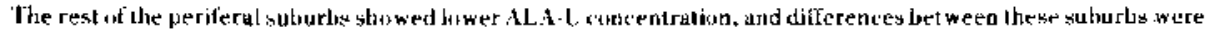

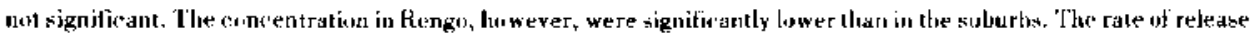
of ALA-li is exessively high in central Santiagu and San Btenardo. We llerelore suggest stricter controls over le ad endissions by incour velic les, as well as from industrial sinurces.
\end{abstract}

Bajo el término de "smog", se esconde una gran variedad de sustancias, unas más dañinas que otras. Existen gases. líquidos y particulas sölidas. Los gases más comunes son el $\mathrm{SO}_{2}, \mathrm{CO}$ y algunos oxidantes, el resto sólo se estudia, en nuestro medio, como polvo en suspensión y contiene tóxicos que presentan un riesgo para la población, tales como los metales pesados y entre ellos es particularmente tíxico el plomo.

El plomo es agregado como antidetonante a la bencína de Ins automóviles, a razín de 0,5 gr., por litro de carburante en forma de tetra y trietilo de plomo. Las fundiciones y fábric as de baterías liberan a la atmósfera grandes cantidades de este metal en forma de aerosol. 1.2,

Su efecto tóxico sobre los mamíferos se debe a la inhibición de varias enzimas que participan en la sintesis del hem en los eritroblastos de la médula ósea. Una de ellas es la delta-ALA dehidrogenasa, su inhibición trae como consecuencia una acumulación del ácido delta amino levulírico en la sangre y su consiguiente eliminación en la orina (ALA-U), donde puede ser determinado. Su eliminación aumenta de manera exponencial con respecto al alza de concentración de plomo en la sangre..3.

Laboratorio de Ecologia, Institulo de Ciencias Biolígicas. Pontificia Universidad Católica de Chile.
El ALA-U, es utilizado corrientemente en medicina ocupacional como un test de exposición precoz en la intoxicación por plomo. (4, 5. 6. 7, 8, 9)

Roels y col. 110.111 , encuentran que los niños son más sensibles a la intoxicación por plomo eliminado más ALA-L que los adultos.

La sensibilidad de este test de exposición a plomo nos ha llevado a utilizarlo conro un indic'ador biológico de la contaminación por plomo en la ciudad de Santiago, comparando áreas periféricas con el centro de la gran ciudad, midiendo el ALA-U en niños cuyas edades fluctúan entre 2 y 5 años. Las mediciones en ellos no se enmascaran por el consumo del cigarrillo, el cuál hace subir los niveles de ALA-U.112!

\section{SITIO DE EXPERIMENTACION}

Santiago se ubica en un valle rodeado por cordones montañosos que confinan el aire en su interior. Los escasos vientos soplan en dirección S., SW y existe una inversión de temperatura tasi constante entre 600 y $1.500 \mathrm{~m}$., notoria sobre todo en invierno.

Los vehiculos de combustion interna constituyen la principal fuente contaminante para el centro de la ciudad, en donde la edificación en altura inmobiliza las capas de aire. Una fuente adicional de 
metal proviene de industrias, ubicadas la mayor parte al $S$. y W' del valle.

Nuestros sectures de muestreo se localizan en las áreas más densamente pobladas y en los cuatro puntos cardinales a unos $10 \mathrm{~km}$. del centro. ellos son:

- Santiago centro.

- Norte. Comuna de Conchalí.

- Oeste. Comuna de Pudabuel.

-Este. Comuna de Las Condes.

- Sur. Comuna de La Cisterna.

Estos puntos aparecen indicados en la figura 2.

Además de estos puntos, hemos medido en San Bernardo a $20 \mathrm{~km}$. del centro hacia el Sur, pero aun al interior del valle y finalmente escogimos otro punto fuera de los cordones montañosos, por lo tanto, fuera de la capa de inversión que allí se genera y se sitúa a $120 \mathrm{~km}$. al Sur en una pequeña ciudad rural, Rengo.

Las mediciones se realizaron todas ellas en los meses de julio y agosto, meses durante los cuales la contaminación atmosférica general es elevada. 13 :

\section{METODO}

El método que hemos utilizado fue descrito primeramente por Wolff(14), y luego por Kunh. 15) La determinación no necesita de una purificación previa. eliminando asi los largos pasos de cromatografia.

El método, posee una buena repetibilidad y es fácil de realizar en el laboratorio.

Las muestras de orina fueron tomadas en niños que viven y asisten a jardines infantiles, en el lugar de muestreo, todas ellas se tomaron entre las 10:00 y las 12:00 horas. En la tabla 1 se muestra el número de jardines muestreados en cada sector. Una vez en el laboratorio, se les midió su densidad y se prepararon con los reactivos para ser medidas en el espectrofotómetro al dia siguiente, debido a la lentitud de la reacción de coloración.

\section{RESULTADOS}

Los resultados vienen expresados en ug ALA-U/ml, orina. En la tabla 1, se muestran los

\section{Tabla N.0 1}

Valores promedios (x), error standard (S ) y número de mediciones (n) de ALA-U de orina de niños en los lugares muestreados y por jardínes.

\begin{tabular}{|c|c|c|c|c|c|c|c|c|c|c|c|c|c|c|c|}
\hline \multirow{2}{*}{$\begin{array}{l}\text { LUGARES } \\
\text { JARDINES }\end{array}$} & \multicolumn{3}{|c|}{ CONCHALI } & \multicolumn{3}{|c|}{ LAS CONDES } & \multicolumn{3}{|c|}{ CENTRO } & LA CISTERNA & \multicolumn{3}{|c|}{ LAS REJAS } & \multicolumn{2}{|c|}{ SAN BDO. RENCO } \\
\hline & $\mathbf{1}$ & 2 & 3 & 1 & 2 & 3 & 1 & 2 & 3 & 12 & 1 & 2 & 3 & 1 & 1 \\
\hline $\bar{x}$ & 6.0 & 7.6 & 9.2 & 6.6 & 7.0 & 8.0 & 13.5 & 11.9 & 16.2 & 5.38 .3 & 6.4 & 5.9 & 5.4 & 14.9 & 4.5 \\
\hline$S_{-}$ & 1.0 & .7 & 1.3 & .7 & 1.2 & .7 & 1.0 & 1.1 & 1.7 & $.4 \quad .4$ & .6 & .9 & .5 & 2.1 & .3 \\
\hline n & 15 & 14 & $\mathrm{I} 4$ & 11 & 15 & 14 & 14 & 14 & 15 & 1427 & 15 & 15 & 15 & 12 & 15 \\
\hline $\bar{x}$ & & 7.5 & & & 7.3 & & & 13.9 & & 7.3 & & 5.9 & & 14.9 & 4.5 \\
\hline
\end{tabular}

valores promedios provenientes de cada jardín infantil y por área, se incluye además el error estándar ( $\mathrm{S} x$ ) y el número de mediciones realizadas (n).

En la figura 1, se muestra el promedio de la concentración de ALA-U por lugar en un plano esquemático de Santiago. El tamaño de los círculos es proporcional al ALA-U medido.

Se puede observar que la orina de niños del centro de Santiago presenta valores de ALA-U mayores que el resto de los lugares medidos, excepto comparado con San Bernardo, el cual presenta también valores muy altos. Para poder determinar si las diferencias observadas en el gráfico eran estadisticamente significativas, se procedió a realizar un análisis de la varianza, utilizando todos los datos obtenidos, se demuestra así que existen diferencias altamente significativas $(p<0.01)$ entre los lugares muestreados. Comparando los lugares por pares entre sí, mediante el test de Scheffé resultó que el centro presentaba concentraciones significativamente mayores al resto de las estaciones, excepto con San Bernardo, con el cual resultó no ser significativamente distinto. El resto de 


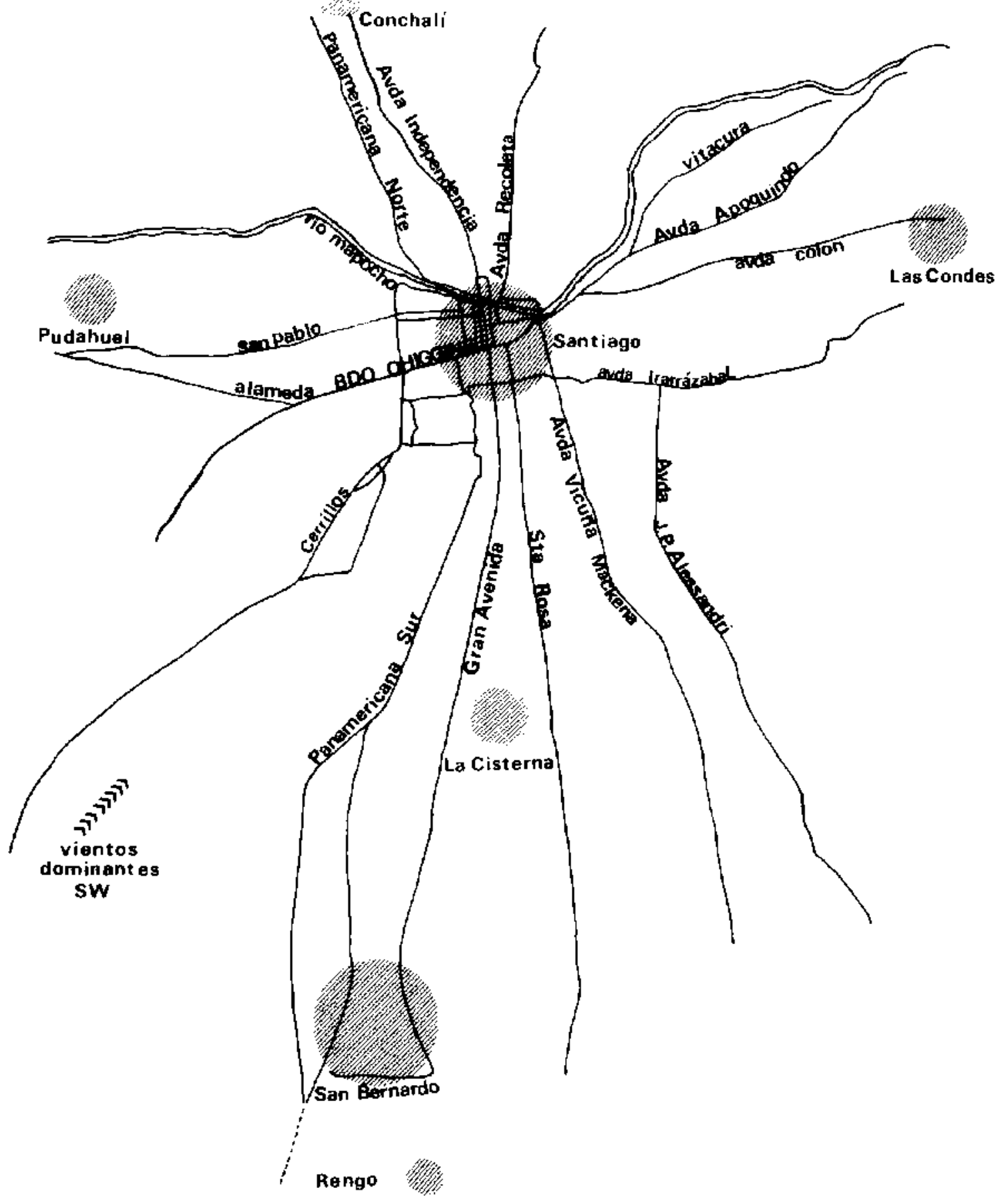

FIG. 1: Ubicación de los lugares muestreados y concentración de ALA-U. El diámetro de los círculos es proporcional a la concentración ( $2 \mathrm{~mm}=\operatorname{lug}$ ALA-U/ml orina). 
los lugares nu muestran diferencias significativas. Debemos aclarar que el test de Scheffé es muy poes sensible y por lo tanto es muy fácil cometer un error estadístico del tipo II, o gea, aceptar la hipcitesis nula cuando ésta es verdadera, Esto nus llevó a utilizar el test de los contrastes ortogonales más discriminalorio. el cual exige el mismo número de medidas para cada estaciön de muestreo. Escogimus para ello, 11 mediciones al azar por lugar y realizamos los contrastes correspondientes. En el primero de ellos. comparamos San Bernardo con Santiago centro, según el test empleado estos 2 lugares entre si no presentan una diferencia significativa $(p>0.05$ ). Esta diferencia es significativa cuando se comparan estos 2 lugares con el resto $(\mathrm{p}<0.05)$. Las distintas comunas del Gran Santiago no muestran diferencias entre ellas $(\mathrm{p}>0.05)$. pero sí son significativamente distintas $(p<0.05)$ a las muestras de Rengo.

\section{DISCUSION}

\section{El método}

Como ya dijéramos en la introducción, el ALA-U ha sido utilizado como test precoz de contaminación por plomo. Una de las mayores dificultades de este tipo de estudio se refieren, a la determinación del ALA$\mathrm{U}$, para ello se han utilizado hasta ahora, técnicas complicadas que incluyen paso por resina ${ }^{\prime(t)}$ y mediciones espectrofotométricas. El método que nos(-) tros hemos utilizado, logra medir el ALA-U de manera precisa, rápida y simple, eliminando las interferencias gracias a una doble determinación con y sin acetil-acetona. Con este método, se puede trabajar hasta 20 muestras en una sola tarde.

Otra de las dificultades en la medición de ALAL, la presenta la variación en la concentración misma de la orina, que varía a lo largo del día y depende de los alimentos líquidos consumidos. Para obviar este problema, muchos autores han medido, ${ }^{10.11]}$ junto con el d-ALA, la cantidad de creatinina cuya liberacion, es muy constante para expresar luego la cuncentración de d-ALA con respecto a creatinina. Para nusotros esta manera de proceder presenta serias dudas, ya que la liberación ded-ALA también es muy constante y nada permite asegurar que la eliminación de las dos sustancias se realice en la misma o distinta fornia, Legando a ellmascararse sus variaciures. La literatura consultada asevera nuestra afirnatión. Lis así comon framer \& Selander ${ }^{\text {din }}$ encuentran una correlarioin al amente significativa entre las concentraciones de Al, A-li y creati- nina, por lor cual recomiendan la expresion ug ALAliml de urina.

Todo ello. hizo que nosutros tomásemos sólo la concentración de d-ALA, muestreando la orina siempre a la misma hora y aportando un factor de corrección proporcionado por la densidad de la orina ${ }^{i \mid \boldsymbol{s})}$

En las muestras medidas, a pesar del factor de corrección, se observa una gran variación individual que se puede apreciar en el error estándar de cada medición, ello bizo que tomásemos un gran número de muestras; de esta manera, la variabilidad intergrupo sería testada contra la varianza residual que incluye las variaciones individuales, con un alto número de grados de libertad.

A la luz de los análisis estadísticos realizados, podemos inferir que existirian 2 focos de contaminación par plomo de los puntos medidos por nosotros. éstos aparecen claramente representados en la Fig. l y corresponden al centro de Santiago y San Bernardo. Las fuentes emisoras en ambos puntos son distintas: mientras en el centro de Santiago el plomo liberado proviene principalmente del gas de escape de los automóviles, en San Bernardo serían las industrias las responsables de las altas concentraciones de Pb atmosférico. Entre las industrias que liberan en gran cantidad este metal, existen en San Bernardo: una fábrica de baterias y una fundiciin de aceros, una fábrica de carburos y la maestranza de Ferrocarriles.

El resio de las estaciones, exceptuando a Rengo, presenta concentraciones comparables entre síy no significalivamente distintas. Podriamos afirmar que en estos puntos no existen fuentes locales importantes que lus contaminen y por to tamio, su concentración depende de los aportes que realicen los focos antes mencionados. Si a ello sumamos la influencia de los vientos dominantes, encontramos explicable que en Pudahuel existan las concentraciones más bajas $(5.9 \mathrm{hg} / \mathrm{ml})$ y que le siga La Cisterna. ya que esta comura se encuentra alimentada por P'b proveniente de San Bernardo. En cuanto a Las Condes y Conchali, reciben la intluencia directa de Santiago centro por los vientos y presentan entunces. concentraciontes comparathes antre si.

Si comparamos entre si los jardines infantiles medidos al interior de cada uno de los lugares, encontramos que sólo con excepción de La Cisternas no existe una diferencia significativa al interior de cada grupo. Esto nos estaría indicando yue la concentración de plomo en esos lugares es similar para un área de algunos kilometros cuadrados $(30-60)$ 
$\mathrm{km}^{2}$ ). Este comportamiento podrá variar debido a la existencia de fuentes industriales muy localizadas, a partir de las cuales la contaminación por metales pesados decrece en forma exponencial con la distancia. "I Las dos muestras medidas en La Cisterna difieren signiticativamente entre sí. ya que una de ellas recibe el apurte de una fuente de tipo industrial. este fenómeno explicaria la gran variabilidad individual detectada en San Bernardo, cuyos valores presentan una amplitud de 23,6 ug/gr ALA-U.

Rengrt se ubica fuera del valle de Santiago y sus concentraciunes son significativamente menores al resto. Esta diferencia no es elevada (p 0.05 ) debido seguramente a la influencia que puede presentar sobre esta cindad la fundicioin de Caletones que. como toda fundición. libera grandes cantidades de metales pesadis. 11

Según Lauwerys'19, on un sujeto normal la excreción de ALA-U no debiera sobrepasar 4,5 $\mathrm{ug} / \mathrm{ml}$. Una concentración de $20 \mathrm{ug} / \mathrm{ml}$. es un indice de absurcion exresiva de plomo. Si calculames la frecuencia relativa de nuestras mediciones en porcentaje. podenos observar que el mayor porcentaje de valores (80-90\%) se ubica entre $0-10 \mathrm{ug} / \mathrm{ml}$., para lus lugares poco contaminados, no asi para Sanliag" centro en donde las mediciones en su mayoría $(70 \%)$ se sitúan entre 10 y $20 \mathrm{ug} / \mathrm{ml}$., habiendo un $14 \%$ de ellas sobre $20 \mathrm{ug} / \mathrm{ml}$., o sea que presentan una absorcion excesiva de plomo, algo similar acurre en San Bernardo con el 58\% de valores entre 10 y $20 \mathrm{ug} / \mathrm{ml}$ y un $16 \%$ sobre 20 $\mathrm{g} / \mathrm{ml}$. En Rengo, en cambio, es un area poco contaminara y el $100 \%$ de los niños medidos tiene valores bajos $10 \mathrm{ug} / \mathrm{ml}$. Esto nos está indicando que nuchos niñus están absorbiendo en furma exresiva el $\mathrm{Pb}$ en el Gran Santiago y sobrepasan las dosis consideradas como normales.

La concentrarión de ALA-L' la hemos utilizado como un indicador biológico de la contaminación por plomu, llegado a los niños pur vía aéréa. La concentracion de plomo que cuntiene el agua porable es muy baja y la diferencia que presentan entre sílas distintas plantas de tratamiento, son aun menures.

En cuanto a la alimentación que constituye otra vía de llegada de plomo a los niños, no existiría ninguna razion para pensar pue determinado sector estaría recibiendo más plomo que otro isobre toxdoen frutas y verdurasi a través de esta vía. Todo ello. nos hace pensar que las variaciunes que se observen en la eliminación de ALA-U dependerán del plumı absorbid, por via pulmonar, o sea, el metal presente como contaminante almusforice. Palat Bryce-Smith y col. 201 , la absorción directa de partículas con plomo por inhalación, representa la mayor fuente do contaminación en las ciudades.

Una gran interrogante nos asalta écuáles podrán ser las consecuencias de esta absorción cxcesiva que hemus detectado? Landrigan y col.'2"', han comprobado la disminucion en el CI de 9 purtos de promedio, en niñus expuestos a mayores roncentraciones de plomo atmosférico, encontrando incluso que los niñus presentaban mayor torpeza, comparado con un grupo no contaminado, en trabajos molrices finos.

Las investigaciones de Bryere.Smith y col.rzor aportan otrós datos aún más alarmantes, ya que st:gún estos autores, los niveles llamados actualmente "normales" están asociados con problemas de inteligencia, velocidad de aprendizaje, hiperactividad. Si a esto le sumamos el plomo que puede atravesar la placenta y afectar el SNC del fetu. nosotros al igual que eston autores, proponemus que se legisle rápidamente para evitar la adic ión de f'b al carburanle de los vehiculos.

\section{RESUMEN}

Sc midió el contenido en ácido delta amino levulínico (ALA-U) en orina de niños de 2 a 5 años de edad. en jardines infantiles del centro y periferia de Santiago y en Rengo. La concentración de ALA.U, se determinú por espectrofotometría a $\mathbf{5 5 3} \mathrm{nm}$. según la técnica de Wolff. Los resultados muestran gue las áreas mas contaminadas son el centry de Santiago y San Bernardo, las comunas medidas no presentan diferencias cstadisticamente significalivas entre si. pero sus valores son superiures a los que muestra el lugar menos contaminado, Rengo. La liberación de ALA-U es excesiva en el centro de Santiago y San Bernardo, la que nos hace sugerir un mayor control subre las emisiones de plomm, tanto por parte de los vehículos, como por fábricas y fundiciones.

\section{AGRADECIMIENTOS}

Los autores agradecen el apoyo dado por la Direccion de Investigación de la Pontificial Universidial Católica de Chile. Proyertos DIUC 94/78.

\section{REFERENCIAS}

\footnotetext{
1 Desueger de Smet, S'. D Duvignenud, P. "Accumulation de mélaux lourds toxigues dans divers ecosystemes terrestres pollues pas des retombées d"origine industriele"., Bull. Sar:. Roy. But. Bel. 107: 147, 1974.
} 
2 Djuric, D.; Graovat-Leposacit, $L$. Milie, S, "Eraluation of lead smelter filcer system by a bioassay un expused population". Int. Arch. Occup. Envirion. Health 35: 241. 1975.

"Selunder, S. "Interrelationship Between Lead in Bloud, Lead in Urine, and ALA in Urine During Lead Work". Brit. J. Ind. Mell. $27: 28.1970$.

${ }^{4}$ Danieli, G.; Gajulos-Torok, M. \& Gajdos, A. "Ln nulveau test bjulugique du saturnisme. Augmentation du taux urinaire et plasmatique de l"acide delta aminolévulinique". Path. Biol. 9: [48]. 1961.

jBasin, $\boldsymbol{B}$. "Le dogage de l'acide delta-aminolevulinjque dang le satumisme". Arch. Mal. Prof. 24: 638, 1963.

"Cranter, $K$ Selander, S. "Studies in Lead Poisoning, Comparisun Bet ween Diflerent Laboralon Tesls. Brit. J. Ind. Med. 22. 311. 1965

¿ Robinson, T. R "Delta Aminolevulinic Acid and Lead in Urine of Leud Antiknock Workers". Arch. Environ. Health 28: 133. 1974.

${ }^{8}$ Tomokuni. $\boldsymbol{K}$. "Delta Aminolvulinic Acid Dehydratase Test for Lead Exposure". Arcb. Environ. Health 29: 274. 1974.

${ }^{9}$ Luyche, D,; Rooseis, D.; Bossirov, J. M. W van Assche, $F$. "The Use of the Urinary Excretion of Delta Aminulevulinic Acid as a Criteriun for Lead Absortion in Industrial Medicine and Insurance Medicine". Int. Arch. Occup. Environ. Health 39 : 191. 1977.

10 Roels, H. A., Lutwerys, R. R ; Buchet, J. P. y cols. "Response of f'ree Arythrocyte Purphyrin and Urinary Delta-aminolevulinic Acid in Men and Women Moderately Exposed to Lead". Int. Arch. Arbeitsmed 34: 97.1975.

" Roels, H. A.; Buchet, J. P.; Lauuerys, R. R. y cols. "Impact isf Air Pollution by Lead on the Heme Biasynthetic Path-way in
Schuol-age Children". Arch. Environ. Health 31:310. 1976.

12 Salle, H.J. \& Zielhuis, R. L. "Influence of Smoking on Anioolevulinic Acid Dehydratase Activity, Haematocrit and Lead in Blood in Adult Urban Women". Int. Arch. Occup. Eqviron. Health 40: 111, 1977.

13 Prugrama para el Control de la Cuntaminación Atmosférica en el Area Metrupolitana de Santiago. INTEC-CORFO, SNS. Santiago, Chile. 164 pp.

14 Wolff, F. C. Método simplificado para la determinación del ácido delt a smino levulínico como indicadur biológio de intoxicación plúmbica. Revista Médjca de Chile 102: 227, 1974.

15 Kuhn, G. “Autonated analysis of Delta-aminolevulinic Acid in Crine". Int. Arch. Occup. Environ. Health 40: 37. 1977.

16 Williams, M.K.\& Few, J. D. "A Simplified Procedure for the Determination of Urinary d-aminolerulinic Acid". Brit. J. lnd. Med. 24: 294, 1967.

12 Cramer, K. \& Selander, S. "Control of Lead Wurkers by Determination of Urinary d-aminolevulinic". Brit. J. Ind. Med. 24(4): 283. 1967

18 Elkins, H. B.; Busnotto, L. D. \& Richmond. M. "The Osmo iurity Adjusanent in Urine Analysis". J. Oceup. Mexd. 8: 528. 1966.

19 Lawwerys, $R$. "Precis de Toxicologie Industrielle et Desintoxications Profesionelles". Bélgica. Editorial Buculot. 1972.

20 Bryce-Smith, D.: Mathewes, J. \& Stephens, B. “Mentel Heallb Effects of Lead on Children". Ambio 7(5-6): 192. 1976.

${ }^{21}$ Landrigan, P. J.; Whitworth, R. H., Batoh, R. W.; Staehling, N. W, Borthel, W. F, \& Alosenbium, B. R. "Neuropsychological Dysfunction in Children with Chronic Low Level Lead Absorption". Lancet 4(7909): 708, 1975. 